Humuskolloiden bereits durch Fickendey ${ }^{7}$ ), wie weiter durch den einen von uns festgestellt worden $\operatorname{sind}^{8}$ ).

Was die Humussäure anbelangt, so findet man sie im Wasser von Torfstichen, wie überhaupt in allen aus Moorbildungen ihren Ursprung nehmenden Gewässern fertig vorgebildet, wie dies deren braune Farbe und sonstigen Eigenschaften beweisen. Will man sie herstellen, so genügt es, eine humusreiche - also an organischer Substanz reiche - Ackererde, noch besser, einen Niederungs- oder kultivierten Hochmoorboden mit verdünnter Salzsäure zu digerieren und dann mit destilliertem Wasser auszuwaschen. Man entfernt so die Kalk- und sonstigen Salze, welche auf 'das Humuskolloid koagulierend wirken, und erhält nach Fortwaschung der Salzsäure ein lichtbraun gefärbtes Wasser, das ohne weiteres in der hier in Frage kommenden Richtung Verwendung finden kann. Für Herstellung weiterer Humuspräparate vergleiche man z. B. Schloesing's Angaben ${ }^{9}$ ).

Es sei nochmals darauf hingewiesen, daß, wie bereits $S \mathrm{chübler}$ hervorhob, durch Alkali

7) Journ. f. Landwirtsch. 54, 343 (1906)

8) Koll.-Zeitschr. 3, 199 (1908). $(1874)$.

9) Annales de chimie et de physique, Série 2, 532 gelöstes Humuskolloid nicht zur Herstellung von kolloidem Gold nach der erwähnten Methode brauchbar ist, während Zusatz von sehr wenig verdünnter Alkali- bezw. Alkalikarbonatlösung $z u$ in Reaktion begriffener Gold- und Humussäurelösung die Bildung des Goldkolloids etwas $\mathrm{zu}$ beschleunigen scheint.

In Anschlub an diese Mitteilung sei noch ein anderes Zitat aus alter agrikulturchemischer Literatur gegeben, das ebenfalls ein Beispiel frühzeitiger Beobachtung eines Kolloidvorganges, der Kataphorese, ist. Sprengel sagt 1844 in seiner "Bodenkunde" ${ }^{10}$ ): "Setzt man humussaure Salze, in Wasser gelöst, dem Strome der voltaischen Säule aus, so entsteht sogleich eine Zersetzung derselben. Die Humussäure zieht sich nämlich in braunen Flocken um das positive oder Zinkende zusammen, während die Basen sich um das Kupfer- oder negative Ende der Polardrähte ansammeln." Humussaure Salze in Wasser gelöst sind, mit anderen Worten, durch Alkali dispergiertes Humuskolloid.

10) Bd. 1, 220 (1844). Mir nìcht zugängliche Arbeiten Spreng el's geben die gleichen Mitteilungen sogar schon im Jahr 1826 . Vgl. Ba umann, Geschichte der Humussäuren, Mitteilungen der Kgl. Bayr. Moorkulturanstalt 1909, Heft 3.

\title{
Ueber die Viskosität und die Koagulation von Kautschuklösungen.
}

\author{
Von H. W. Woudstra.
}

Seit einiger Zeit bin ich beschäftigt, den Koagulationsprozeß kolloider Lösungen mittels der Methode der inneren Reibung zu studieren.

Ich habe bereits am $\mathrm{Muthmann'schen}$ Silbersol eine Reihe Viskositätsbestimmungen gemacht ${ }^{1}$ ).

Diese Bestimmungen wurden vorgenommen an Mischungen von Sol und Salzlösungen; die Quantität letzterer war so bemessen, daß die Koagulation sehr langsam stattfand. Es wurde gefunden, daß die innere Reibung allmählich sank. Dies Ergebnis ist sehr merkwürdig. Weitere Untersuchungen wurden an anderen kolloiden Lösungen angestellt und haben ähnliche Resultate ergeben.

$\mathrm{Da}$ meine Arbeit noch nicht zu einem AbschluB gebracht ist, werde ich nur meine Ergebnisse mit einer kolloiden Kautschuklösung

1) Chem. Weekblad 303 (1908). Zeitschr. f. phys. Chem. 63, 619 (1908). Koll.-Zeitschr. 3, 233 (1908). mitteilen. Wie ich im Aprilheft dieser Zeitung sehe, sind nämlich P. Schidrow it $z$ und $H$. A. Goldsbrough auch mit der Untersuchung von Kautschuklösungen beschäftigt und es wird vielleicht von Interesse sein, meine Arbeit vorläufig zu publizieren.

Anfangs war meine Absicht, die Viskosität einer Kautschuklösung zu bestimmen und ihren Verlauf während des Alterns der Lösung $z$ u beobachten. Da die Eigenschaften von Kolloiden in hohem Maße von ihrer Vorgeschichte abhängig sind, kam mir später der Gedanke, Kautschuksorten verschiedener Herkunft, Alters und Bereitungsweise im gleichen Lösungsmittel $z u$ gleichstarken Lösungen $z u$ lösen und deren Viskosität $z u$ bestimmen. Es würde sich dann herausstellen müssen, ob der Kautschuk auch in dieser Hinsicht zu den Kolloiden gehörte, und für die Praxis würde sich vielleicht eine Methode ergeben, welche es ermöglicht, aus der inneren 
Reibung der Kautschuklösungen auf deren Herkunft und Qualität zu schließen.

Was die Zugehörigkeit des Kautschuks zu den Kolloiden anbetrifft, so beziehen sich die Argumente, welche zugunsten dieser Auffassung sprechen, hauptsächlich auf die Eigenschaften des Latex. Soweit mir bekannt, ist ein Kolloidcharakter an den "Lösungen" des Kautschuks niemals eingehend erforscht. Das Fehlen des Diffusionsvermögens durch eine Pergamentscheidewand, welche für das echte Kolloid chrakteristisch ist, findet man, wie sich aus meinen Versuchen ergab, ebenfalls beim Kautschuk. Ich dialysierte während drei Wochen eine Lösung von Rohkautschuk in Toluol, welche durch Glaswolle filtriert war, in einer Dialysierhülse gegen reinen Toluol. Das Dialysat wurde destilliert und hinterließ nur eine Spur Rückstand. Das gleiche Resultat lieferte die Dialyse einer "Lösung" in Schwefelkohlenstoff. Der Rückstand der Destillation des Dialysats im letzteren Falle waren einige Tröpfchen eines gelbbraunen Oels. Nach einigen Tagen kristallisierte dasselbe. Die braunen Kristalle hatten einen sehr eigentümlichen Geruch. Die Verbrennung auf einem Platinspatel lieferteSchwefeldioxyd. Vielleicht war diese Substanz eine Verunreinigung des Schwefelkohlenstoffs. Die "Lösung" des Kautschuks in Toluol läßt sich schnell filtrieren durch Glaswolle, aber nur sehr langsam durch Papier. Eine vollständig klare Lösung wurde im Thermostaten auf $25^{\circ}$ gebracht. Nach einigen Tagen fing die Lösung an $z \mathrm{u}$ opalisieren und schlieblich setzte sich eine flockige Fällung zu Boden. Man kann

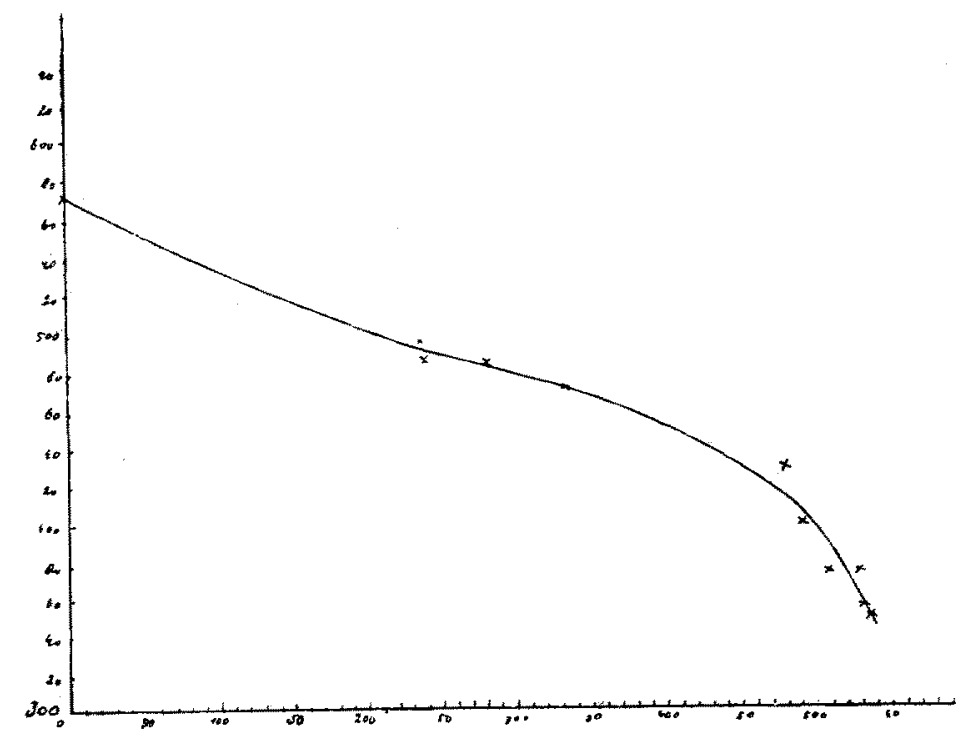

dieselbe Fälung hervorrufen innerhalb kurzer Zeit, wenn man die Temperatur erhöht. Schon bei $+36^{\circ}$ tritt eine deutliche Trübung auf.

Dies Verhalten ist also anscheinend analog der Temperaturkoagulation einer kolloiden Eiweißlösung.

Zum Studium dieses Vorganges mittels der Viskositätsmethode stellte ich "Lösungen" des Rohkautschuks her in Xylol, Tetrachlorkohlenstoff und Toluol, annähernd von gleicher Konzentration etwa $1 \mathrm{~g}$ in $200 \mathrm{ccm}$.

Diese Lösungen wurden durch Filtrierpapier filtriert, welche Operation verschiedene Tage dauerte (Glaswolle war weniger geeignet) und im Thermostaten auf $25^{\circ}$ gebracht.

Das Tetrachlorkohlenstoffsol des Kautschuks war sehr unbeständig; stark opaleszierend, trübte es sich nach einigen Tagen und wurde vorläufig nicht weiter untersucht. Am Xylolsol und am Toluolsol aber wurden innere Reibungsbestimmungen vorgenommen.

Soweit mir bekannt, hat nur Axelrod ${ }^{2}$ ) Viskositätsbestimmungen an Kautschuklösungen angestellt. Er arbeitete mit Lösungen in Benzol und in Benzin. Der von ihm verwendete Apparat hat im Prinzip grobe Aehnlichkeit mit dem Engler'schen Viskosimeter für Schmieröle. Er arbeitete mit konzentrierten Lösungen. Ich bestimmte die innere Reibung mittels der Ostwald'schen Viskosimeters im durchsichtigen Thermostaten. Da es nicht meine Absicht war, absolute Viskosität zu bestimmen, habe ich nur die Ausflubzeiten gemessen. Die Lösungen befanden sich in Glasröhren mit sorgfältig eingeschliffenen Stöpseln, welche obendrein mit einer Gummikappe versehen waren. Die Dichtigkeit der Lösung habe ich konstant angenommen.

Die innere Reibung der Kautschuklösungen nimmt mit der Zeit allmählich ab. Dies ergab sich aus den mit bestimmten Intervallen gemessenen Ausflubzeiten. In nebenstehender Figur sind die Ergebnisse am Toluolsol des Kautschuks graphisch dargestellt.

Die Versuche am Xylol- und am Tetrachlorkohlenstoffsol sind noch nicht abgeschlossen.

Kön. Marine-Institut Helder.

2) Gummizeitung 19, 1053 (1905). 